\title{
Modèle empirique 3D : application à la gestion des activités de dragage à l'embouchure de l'Adour
}

\author{
Didier RIHOUEY(1),Jean DUBRANNA(2)
}

(1) Responsable transfert technologique, (2) Doctorant

LaSAGeC2/UPPA, 1 allée du parc Montaury, 64600 Anglet

\begin{abstract}
Résumé :
Le développement d'outils prévisionnels d'évolution bathymétrique est une problématique majeure en terme de gestion des activités de dragage. Cette étude présente un modèle morphodynamique empirique, dans lequel les évolutions tridimensionnelles de la bathymétrie sont prises en compte par analyse factorielle et reliées linéairement aux forçages climatiques et anthropiques. Le modèle, qui est ensuite appliqué à l'évolution de l'embouchure de l'Adour, représente correctement les évolutions (ensablement et dragage) du système fosse de garde / chenal de navigation. Les capacités prédictives du modèle, ses limitations et les perspectives de développement sont analysées et discutées. Enfin, l'utilisation du modèle en temps qu'outil d'aide à la décision est testée via la simulation de différents scénarios.
\end{abstract}

\section{abstact :}

The development of tools to predict bathymetric evolution is a major factor in good dredging management. This study presents an empirical morphodynamic model based on eigenfunction analysis, linked linearly to climatic and human forcings. Applied to the Adour river mouth, the model represents well the silting and the dredging of the navigation channel and preventive trench. The accuracy, limitations and possibilities of this approach are analyzed and discussed. Finally, the model is used as a dredging management tool by simulating different scenarios.

Mots clefs :

Modèle empirique, Analyse factorielle, Dragage, Adour, Aide à la décision

Key words :

Empirical model, Eigenfunction analysis, Dredging, Adour, Management tools 


\section{Introduction}

Préserver une bonne accessibilité aux installations portuaires reste l'un des problèmes majeurs qui se pose aux gestionnaires des ports. L'objectif principal est de maintenir un chenal de navigation suffisamment profond pour permettre aux navires marchands d'accéder aux quais. Certains ports disposent de systèmes de dragage propre, leur permettant d'intervenir à tout moment. D'autres, comme le port de Bayonne ne disposent pas de moyens propres et doivent faire venir des dragues afin de maintenir leurs chenaux de navigation. Pour ces ports, le problème est plus complexe car une brusque remontée des fonds peut entraîner la fermeture du port durant plusieurs jours, et donc des pertes financières importantes.

L'amélioration des connaissances et le développement d'outils prévisionnels d'évolution bathymétrique sont donc devenus des problématiques majeures et stratégiques en terme de gestion des activités de dragage. On distingue deux grandes catégories de modèles d'évolution morphodynamique : les modèles numériques basés sur les équations fondamentales de la dynamique, les modèles empiriques basés sur l'analyse statistique de données in-situ de morphologie et des forçages. Ce dernier type de modèle constitue l'objet de cet article.

Les modèles empiriques sont déjà largement utilisés par les météorologues et les océanographes, qui disposent d'un grand nombre de données in-situ par le biais des stations d'observation et de la télédétection. En ce qui concerne la morphodynamique côtière, le manque de mesures bathymétriques couplées à des mesures de forçages climatiques et hydrodynamiques, a retardé l'application de ces modèles. Cependant, l'utilisation de l'analyse factorielle couplée à un modèle linéaire a été validé pour décrire les évolutions morphologiques de plages sableuses exposées aux vagues (Aubrey, 1980; Hsu et al., 1994; Rihouey, 2004) $\mathbf{2 , 6 , 8}$. Les méthodes d'analyse factorielle (AFC), couramment utilisées pour caractériser l'évolution morphodynamique des plages sableuses (Rihouey, 2004), se sont également révélées efficaces pour étudier la dynamique des estuaires (Cuadrado et Perillo, 1997 ; Horrillo-Caraballo et al., 2002 ; Maron et al., 2004) $)^{4,5,7}$.

Cette étude présente un modèle morphodynamique empirique, dans lequel les évolutions tridimensionnelles de la bathymétrie sont pris en compte par AFC et reliés ensuite linéairement aux forçages climatiques et anthropiques. Le principe de l'AFC et la formulation utilisée pour reliér les évolutions bathymétriques aux forçages climatiques et anthropiques, sont présentées dans la section 2. Le modèle est ensuite appliqué à l'évolution de l'embouchure de l'Adour dans la section 3. Les capacités prédictives du modèle, ses limitations et les perspectives de développement de la méthode sont analysées et discutées dans cette section. Enfin, les capacités du modèle en temps qu'outil d'aide à la décision sont testées dans la section 4. 


\section{Principe du modèle empirique 3D}

\subsection{Prise en compte des évolutions tridimensionnelles de la bathymétrie}

Les méthodes d'analyse factorielle permettent une forte compression des données et de facilitent l'analyse de jeux de données nombreuses et complexes. Le principe de la méthode 2D consiste à exprimer la profondeur $h\left(\xi_{l}, t_{k}\right)$ par des combinaisons linéaires de produits de fonctions (ou modes) spatiales et de fonctions (ou modes) temporelles.

Considérons un échantillon de $K$ surfaces du fond comportant chacune $L$ profondeur $h\left(\xi_{l}, t_{k}\right)$, où les $\xi_{l}$ représentent les points sur une grille régulière, avec $1 \leq l \leq L$ et $1 \leq k \leq K$. Ainsi, l'objectif de l'AFC est d'exprimer les données sous la forme :

$$
h\left(\xi_{l}, t_{k}\right)=\sum_{p=1}^{L} C_{p}\left(t_{k}\right) \cdot e_{p}\left(\xi_{l}\right)
$$

où $e_{p}$ sont les vecteurs (ou modes) propres de la matrice des corrélations $A$ et $C_{p}$ les modes temporels correspondant. Les coefficients de $A$ sont donnés par :

$$
a_{m n}=\frac{1}{L \cdot K} \sum_{k=1}^{K} h\left(\xi_{m}, t_{k}\right) \cdot h\left(\xi_{n}, t_{k}\right)
$$

$A$ est une matrice $\mathrm{L}^{*} L$ symétrique réelle. Elle est donc diagonalisable dans $\mathfrak{R}$, possède L valeurs propres $\lambda_{p}$ avec $1 \leq p \leq L$ et les vecteurs propres correspondant aux valeurs propres $\lambda_{p}$ satisfont l'équation matricielle suivante :

$$
A e_{p}=\lambda_{p} e_{p}
$$

les coefficients $C_{p}$, ou modes propres temporels, sont donnés par :

$$
C_{p}\left(t_{k}\right)=\sum_{p=1}^{L} h\left(\xi_{l}, t_{k}\right) \cdot e_{p}\left(\xi_{l}\right)
$$

Généralement, les 2 ou 3 premiers modes représentent plus de $99 \%$ des données initiales et suffisent pour décrire l'évolution de la bathymétrie. Le premier mode spatial représente la bathymétrie moyenne au cours de la période d'étude. Le mode temporel associé $C_{l}(t)$ représente les variations de la profondeur moyenne. Les modes d'ordre supérieur représentent quant à eux les variations par rapport à la morphologie moyenne et sont liés aux zones où les variations bathymétriques sont les plus importantes. 


\subsection{Formulation du modèle empirique}

Les caractéristiques morphodynamiques d'un estuaire dépendent d'un certain nombre de facteurs parmi lesquels son bassin versant (taille, qualité hydrologique et géologique), la zone géologique de son implantation, ses caractéristiques hydrodynamiques (marée, houle, débit des affluents), les aménagements effectués (ports, digues, barrages, dragages, entretiens des chenaux),... Chaque estuaire présente donc des caractéristiques qui lui sont propres et les forçages à prendre en compte dans un modèle empirique dépendent du type d'estuaire étudié. Dans le modèle ici présenté, les forçages pris en compte sont : les vagues $W$, le débit du fleuve $Q$, et les activités de dragage $D$.

L'évolution d'un estuaire est dépendante de l'état antérieur de sa morphologie et des forçages hydro-météorologiques et/ou anthropiques. Ces forçages possédant un caractère stochastique, l'évolution morphodynamique d'un estuaire peut être décrite comme un processus markovien, défini comme une succession d'états morphologiques interdépendants (Sonu \& James, 1973)9 .

Dans le modèle ici présenté, l'évolution de la bathymétrie est pris en compte via l'utilisation des deux premiers modes temporels issus de l'AFC. $\hat{C}(t)$ et $\hat{C}(t+1)$, qui sont respectivement les valeurs des modes temporels aux l'instant $t$ et $t+1$, sont liés linéairement par la relation suivante:

$$
\left[\begin{array}{c}
\hat{C}_{1}(t+1) \\
\hat{C}_{2}(t+1)
\end{array}\right]=\left[\begin{array}{lllll}
b_{11} & b_{12} & b_{13} & b_{14} & b_{15} \\
b_{21} & b_{22} & b_{23} & b_{24} & b_{25}
\end{array}\right]\left[\begin{array}{c}
\hat{C}_{1}(t) \\
\hat{C}_{2}(t) \\
W(t \rightarrow t+1) \\
Q(t \rightarrow t+1) \\
D(t \rightarrow t+1)
\end{array}\right]
$$

où, $W(t \rightarrow t+1)$ correspond au forçage de houle entre $t$ et $t+1, \quad D(t \rightarrow t+1)$ correspond au forçage de dragage entre $t$ et $t+1, Q(t \rightarrow t+1)$ correspond au forçage de dragage entre $t$ et $t+1$, et où $b_{11}, \cdots, b_{25}$ sont des coefficients ajustés par dichotomie.

\section{$\underline{\text { 3. Application du modèle à l'évolution de l'embouchure de l'Adour }}$}

\subsection{Problématique du site et données utilisées}

Depuis des décennies, l'embouchure de l'Adour présente une tendance continue à s'ensabler et nécessite de coûteux travaux de dragage afin de conserver une profondeur suffisante du chenal d'accès au port de Bayonne. En 1999, les Services Maritimes de la DDE ont décidé de creuser une "fosse de garde" au sud 
de l'embouchure afin de limiter l'ensablement du chenal (figure 1.). Toutefois, même si le phénomène d'ensablement du chenal de navigation a diminué considérablement, deux campagnes de dragage par an restent nécessaires pour maintenir l'équilibre du système fosse de garde - chenal de navigation.

L'utilisation de modèles numériques (Brière, 2005) ${ }^{\mathbf{3}}$ a montré l'existence d'un courant induit par la houle dirigée vers l'embouchure. Ce courant est créé par la présence de la digue Nord qui en créant une zone calme derrière l'embouchure jouxtant la zone agitée des Cavaliers force sa génération. Il a été établi que seuls les évènements suffisamment énergétiques ( $\mathrm{Hs}>4 \mathrm{~m})$, classifiés comme houles d'hiver et tempêtes, génèrent des circulations susceptibles de transporter des sédiments en zone d'embouchure.

\subsubsection{Données bathymétriques}

67 levés bathymétriques ont été effectuées entre décembre 2001 et février 2005 par les services maritimes de la DDE 64 et le $\mathrm{LaSaGeC}^{2}$. L'analyse factorielle requière que l'emprise des données considérées soit de dimension identique. La zone étudiée est donc limitée aux données communes aux 67 levés (délimitée dans sa diagonale par les points $287300 \mathrm{E}, 143850 \mathrm{~N}$ et $287950 \mathrm{E}$, $144100 \mathrm{~N}$ en coordonnées Lambert III (Figure 1.)). 67 Modèles Numériques de Terrain (MNT) ont ensuite été générés à l'aide d'un Système d'Information Géographique (S.I.G.) avec un pas de discrétisation spatiale de $10 \mathrm{~m} * 10 \mathrm{~m}$.

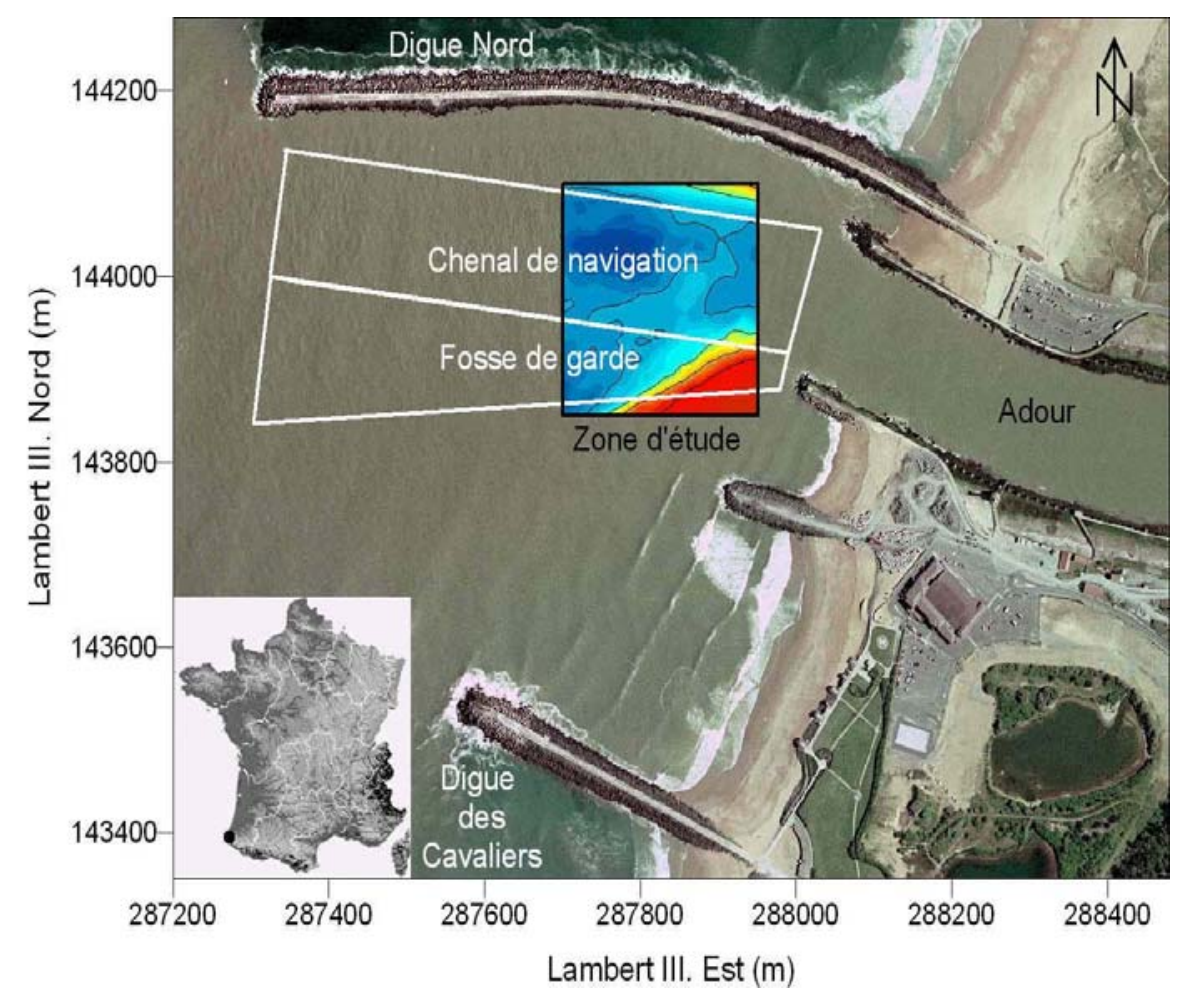


Figure 1. Localisation de la zone d'étude

\subsubsection{Données hydrodynamiques}

Une analyse des paramètres statistiques de la houle pour la zone côtière sud-aquitaine a été réalisée par comparaison de deux jeux de données : une mesure locale sur une période courte et des résultats de simulation du modèle Wavewatch III (www.fnmoc.navy.mil) sur 6 ans et 9 mois (Abadie et al, 2005) ${ }^{\mathbf{1}}$. Cette étude montre qu'il existe une très bonne corrélation entre les hauteurs significatives $H_{s}$ issues respectivement de la mesure et des simulations. Ces données sont donc utilisées pour prendre en compte la houle. Dans le modèle ici présenté, le forçage de houle est considéré proportionnel au nombre de jours où $H_{s}$ dépasse 4,5 et $6 \mathrm{~m}$ entre deux levés bathymétriques.

Les données relatives au débit de l'Adour ont été obtenues auprès de la DIREN. Le débit moyen de l'Adour est d'environ $300 \mathrm{~m}^{3} / \mathrm{s}$ et on relève environ trois crues supérieures à $1000 \mathrm{~m}^{3} / \mathrm{s}$ par an.

\subsubsection{Données de dragage}

Les trajets réalisés par la drague lors des campagnes sont archivés par les services maritimes de la DDE 64. Le forçage relatif au dragage est considéré proportionnel au nombre de passage sur la zone d'étude entre deux levés bathymétriques.

\subsection{Présentation des résultats}

\subsubsection{Résultats de l'analyse factorielle}

67 Modèles Numériques de Terrain ont donc été analysés par analyse factorielle. Les deux premiers modes, qui représentent $99.5 \%$ de l'information initiale, sont présentés sur les figures 2 . et 3 . Les MNT correspondent au modes spatiaux. Les courbes noires correspondent à leur modes temporels associés et les périodes de dragages sont représentées par des traits rouges verticaux.

Le premier mode spatial représente la bathymétrie moyenne au cours de la période d'étude. Le mode temporel associé $C_{l}(t)$ représente les variations de la profondeur moyenne :

- Les fortes valeurs de $C_{l}(t)$ (figure 2.), correspondent à des périodes où la profondeur est plus élevée que la profondeur de la bathymétrie moyenne (situation post-dragage).

- Inversement, les faibles valeurs de $C_{l}(t)$ (figure 3.) correspondent à des périodes où la profondeur est moins élevée que la profondeur de la bathymétrie moyenne (situation ensablée). 


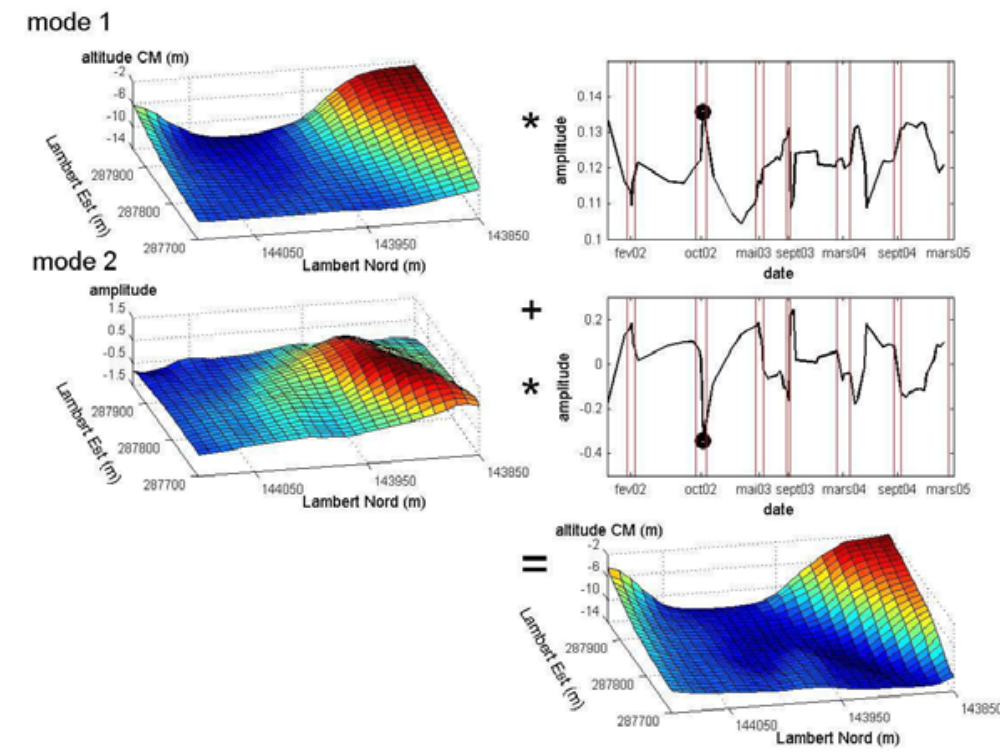

Figure 2. Reconstruction de la bathymétrie à l'aide des 2 premiers modes de l'AFC : morphologie post-dragage

Le second mode spatial représente les zones où les variations bathymétriques sont les plus importantes (ici la fosse de garde). Le mode temporel associé $C_{2}(t)$ représente donc l'évolution de la morphologie de la fosse de garde :

- Les fortes valeurs négatives de $C_{2}(t)$, correspondent à des morphologies postdragage où la fosse de garde est bien creusée (figure 2.).

- Inversement, les fortes valeurs positives de $C_{2}(t)$ (figure 3.) correspondent à des périodes où la fosse de garde est bien ensablée.

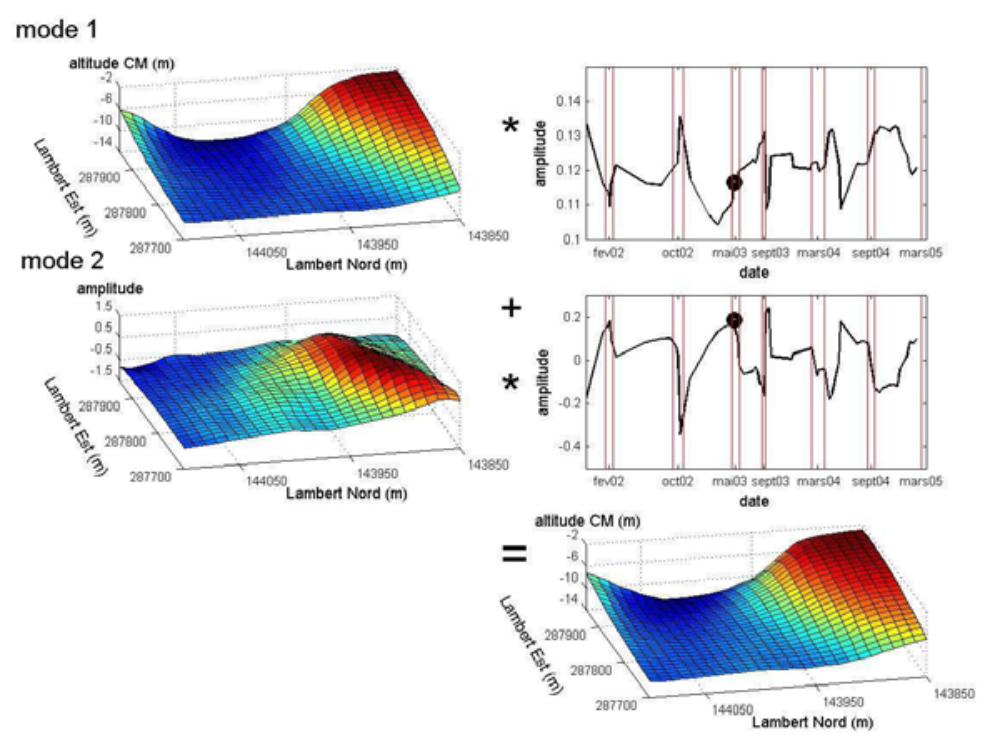

Figure 3. Reconstruction de la bathymétrie à l'aide des 2 premiers modes de l'AFC : morphologie ensablée 


\subsubsection{Résultats du modèle linéaire}

Pour illustrer les résultats de modèle linéaire, seul le second mode est ici présenté (figure 4.). La courbe pleine représente le second mode temporel $C_{2}(t)$ de l'AFC présenté dans la section 3.2.1. La courbe en pointillée représente le second mode temporel $\hat{C}_{2}(t)$ obtenu par le modèle. Les variations croissantes de $C_{2}(t)$ et $\hat{C}_{2}(t)$ correspondent au périodes d'ensablements de la fosse de garde et de l'embouchure. Les variations décroissantes de $C_{2}(t)$ et $\hat{C}_{2}(t)$ correspondent aux périodes de dragage qui sont représentées par des traits rouges verticaux.

- le modèle (courbe pointillée) représente correctement les évolutions (ensablement et dragage) du système fosse de garde / chenal de navigation,

- deux événements ne sont cependant pas représentés par le modèle : des ensablements suivant les dragages de septembre 2003 et mars 2004.

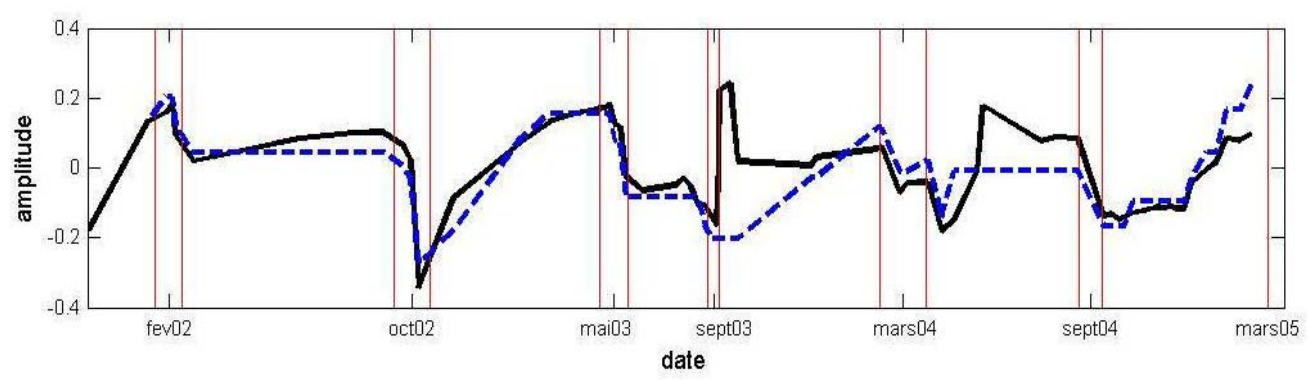

Figure 4. Résultats du modèle linéaire pour $C_{2}(t)$

Les deux événements non représentés possèdent cependant deux caractéristiques singulières :

- on n'observe pas de conditions de houle susceptibles de transporter des sédiments en zone d'embouchure lors de ces deux périodes,

- suite à ces ensablements (représentés par une augmentation rapide de $C_{2}(t)$ ) on observe une diminution de la valeur de $C_{2}(t)$. Or les diminutions de $C_{2}(t)$ ne sont observées que lors des campagnes de dragage.

Ces caractéristiques singulières posent des questions concernant les processus responsables des évolutions bathymétriques de l'embouchure et la qualité de l'information hydrographique. Ces deux problématiques ne sont pas abordés ici mais feront l'objet d'études et de développements complémentaires.

\section{Simulation de scénarios et aide à la décision}

A l'heure actuelle la programmation des campagnes de dragage est réalisée en fonction de contraintes financières et des disponibilités des dragues du G.I.E. (Groupement d'Intérêt Economique) Dragage Port. Ces contraintes imposent la programmation des dates et de la durée de chaque campagne un an à l'avance. 
Cette situation pourrait évoluer dans le cadre de la décentralisation et le nouveau gestionnaire du port sera alors libre de faire appel à d'autres organismes pour réaliser les dragages. Pour optimiser l'exploitation des accès, tout en diminuant le coût des dragages, un certain nombre de questions se posent :

- Une seule période de dragage par an serait elle suffisante?

- Est-il possible d'atteindre un seuil avant d'organiser une campagne ?

- Comment déterminer ce seuil tout en garantissant un accès continu au port ?

Pour répondre à ces questions, un outil prévisionnel d'évolution bathymétrique est nécessaire. Les capacités prédictives du modèle empirique 3D ayant été montrées précédemment, il est alors utilisé pour simuler deux scénarios :

- scénario 1: abandon de la campagne de dragage de mars 2003 et affectation des volumes dragués à la campagne de septembre 2003,

- scénario 2 : abandon de la campagne de dragage de mars 2004 et affectation des volumes dragués à la campagne de septembre 2004.
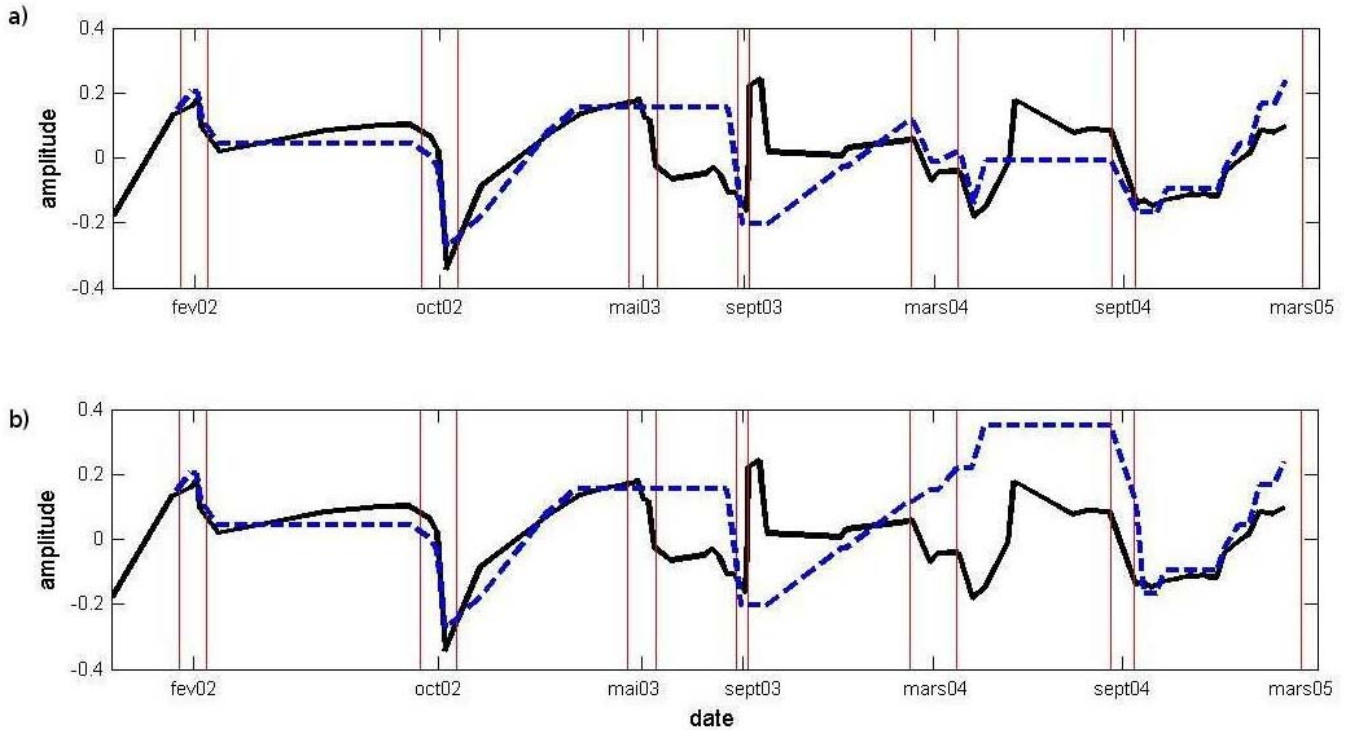

Figure 5. Résultat de la simulation des scénario 1 (a) et 2 (b)

Les figure 5.a. et 5.b. représentent respectivement les résultats des simulations de scénario 1 et 2 . La courbe en noire représente le second mode temporel $C_{2}(t)$ de l'AFC présenté dans la section 3.2.1. La courbe en pointillée représente le second mode temporel $\hat{C}_{2}(t)$ obtenu par le modèle.

La simulation montre que la campagne de mars 2003 aurait pu ne pas avoir lieu. En effet, en mars de 2003 la bathymétrie de l'embouchure présente une morphologie ensablée (fosse de garde et chenal de navigation). La profondeur reste cependant suffisante pour assurer l'accès au port de Bayonne. En l'absence de conditions de houle susceptibles de transporter des sédiments en zone d'embouchure, le modèle 3D prédit que la morphologie n'aurait pas évolué de façon significative entre mars et septembre 2003. Suite à la simulation du report 
du dragage de mars en septembre, la bathymétrie de l'embouchure présente des morphologies de la fosse de garde et du chenal bien marquées.

La simulation montre que l'abandon de la campagne de mars 2004 aurait pu compromettre l'accès au port de Bayonne. En effet, en mars de 2004 la bathymétrie de l'embouchure présente une morphologie faiblement ensablée. Cependant, en présence de fortes conditions d'agitation le modèle 3D prédit un ensablement conséquent de la fosse de garde et du chenal en mars et avril 2005 (valeur importante de $\hat{C}_{2}(t)$ ), La figure 6., qui représente la bathymétrie prédite par le modèle 3D en avril 2005, illustre la situation d'ensablement de la fosse de garde et du chenal de navigation.

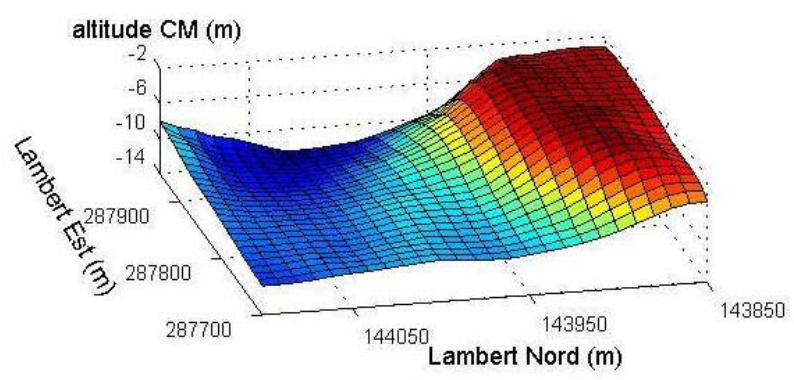

Figure 6. Résultat de la simulation du scénario 2

Les résultats de ces simulations de scénario montrent les capacités du modèle 3D en temps qu'outil d'aide à la décision. A la question « une seule période de dragage par an serait elle suffisante ? » les deux simulations montrent que l'abandon de la campagne de mars peut se révéler hasardeux. En effet, l'analyse des données de houle indique l'existence au printemps de conditions de houle susceptibles de transporter des sédiments en zone d'embouchure. En revanche, au regard des faibles conditions d'agitation estivales, l'abandon de la campagne de septembre pourrait être envisagé. En effet, il est possible d'utiliser le modèle 3D pour déterminer un seuil qui déclencherait (en juin) la programmation de la campagne de septembre.

\section{Conclusion}

La prise en compte des évolutions tridimensionnelles de la bathymétrie par analyse factorielle, reliées linéairement aux forçages climatiques et anthropiques a permis le développement d'un modèle morphodynamique empirique 3D.

L'application de ce modèle à l'évolution de l'embouchure de l'Adour a permis de valider l'approche utilisée. Le modèle représente correctement les évolutions (ensablement et dragage) du système fosse de garde / chenal de navigation. Les capacités prédictives du modèle, ses limitations et les perspectives de développement ont été analysées et discutées. 
Enfin, l'utilisation du modèle en temps qu'outil d'aide à la décision, qui a été testée via la simulation de différents scénarios, pourrait permettre de déterminer un seuil qui déclencherait (en juin) la programmation de la campagne de septembre.

\section{Références}

1 Abadie S., Butel R., Dupuis H., Brière C. (2005). Paramètres statistiques de la houle au large de la côte sud-aquitaine, Comptes Rendus Geosciences, Vol. 337, issue 8, pp. 769-776.

2 Aubrey, D. G. (1980). The statistical Prediction of Beach Changes in southern California. Journal of Geophysical Research, 85(C6), 3264-3276.

3 Brière, C. (2005). Hydrodynamique d'une zone côtière anthropisée : l'embouchure de l'Adour et les plages adjacentes d'Anglet. Thèse de doctorat de l'Université de Pau et des Pays de l'Adour.

4 Cuadrado D. G., Perillo, G. M. E. (1997). Principal component analysis applied to geomorphologic evolution. Estuarine, Coastal and Shelf Science, 44(4), 411419.

5 Horillo-Caraballo, J. M., Reeve, D. E., Li, B. (2002). Eigenfunction analysis of complex estuary morphology. Hydroinformatics 2002, Cardiff, 777-784.

6 Hsu, T. W., Ou, S. H. and Wang, S. K. (1994). On the prediction of beach changes by a new 2-D empirical eigenfunction model. Coastal Engineering, 23, 255-270.

7 Maron P., Rihouey D., Dubranna J. (2004). Méthode d'analyse factorielle appliquée au suivi bathymétrique de l'embouchure de l'Adour, Journées Nationales Génie Civil Génie Côtier, Compiègne, septembre 2004.

8 Rihouey, D. (2004). Analyse statistique de l'évolution morphodynamique des plages sableuses. Application aux sites d'étude du Programme National d'Environnement Côtier et aux plages d'Anglet. Thèse de doctorat de l'Université de Pau et des Pays de l'Adour.

9 Sonu, C. J. and James, W. R. (1973). A Markov model for beach profil changes. Journal of Geophysical Research, 78, 1462-1471. 